\title{
« Equal pay for a similar work ", le mythe régulateur du salaire au poste dans l'Europe de la CECA (années 1950-1960)
}

"Equal pay for a similar work", the regulating myth of job evaluation in Europe at the time of the ECSC (1950s-1960s)

\section{Ferruccio Ricciardi}

\section{OpenEdition}

Journals

Édition électronique

URL : http://journals.openedition.org/travailemploi/5934

DOI : 10.4000/travailemploi.5934

ISSN : $1775-416 \mathrm{X}$

Éditeur

DARES - Ministère du Travail

Édition imprimée

Date de publication : 15 mars 2013

Pagination : 13-24

ISSN : 0224-4365

Référence électronique

Ferruccio Ricciardi, « «Equal pay for a similar work », le mythe régulateur du salaire au poste dans l'Europe de la CECA (années 1950-1960) », Travail et Emploi [En ligne], 133 I janvier-mars 2013, mis en ligne le 01 janvier 2015, consulté le 19 avril 2019. URL : http://journals.openedition.org/ travailemploi/5934; DOI : 10.4000/travailemploi.5934 


\title{
"Equal pay for a similar work ", le mythe régulateur du salaire au poste dans l'Europe de la CECA (années 1950-1960)
}

\author{
Ferruccio Ricciardi $\left.{ }^{*}\right)$
}

À partir d'une étude sociohistorique, cet article interroge le projet de régulation sociale qui était sousjacent à la promotion d'un nouvel outil de gestion du personnel - la job evaluation ou salaire au poste - dans les pays de la Communauté européenne du charbon et de l'acier durant les années 1950-1960. Pour les technocrates européens, l'application de cet outil constitue un préalable technique pour la modernisation du système de la négociation collective sur fond de rationalisation de la main-d'œuvre et de stabilisation des relations professionnelles. L'article montre comment la double "circulation» - transnationale et locale - dont la job evaluation fait l'objet dans le secteur sidérurgique a influencé la conception, l'adaptation, voire la transformation de cette technique. L'inscription de celle-ci dans le jeu de la négociation collective ainsi que dans les processus de mise au point des classifications professionnelles rend compte de sa capacité à répondre ou non aux finalités qu'on lui fixe.

Dans la «boîte à outils» que les «missionnaires» envoyés aux États-Unis grâce aux financements du plan Marshall ramènent en Europe dans les années 1950, les techniques de gestion du personnel occupent une place importante (BolTANSKI, 1981). Si la course à la productivité inaugurée au lendemain de la seconde guerre mondiale devient, avec son corollaire d'outils et de méthodes d'organisation, un principe autonome de régulation politique (MAIER, 1987), c'est aussi en raison des efforts entrepris pour moderniser les relations sociales dans les entreprises (CAREW, 1987). Ces nouvelles techniques de gestion de la main-d'œuvre s'inscrivent dans un mouvement de rationalisation du travail qui, entamé durant l'entre-deux-guerres dans un climat de fortes tensions sociales (le taylorisme, en Europe, se traduisant par l'intensification du travail, la réduction des temps d'exécution des tâches et la dégradation des conditions de travail [MOUTET, 1997]), se tourne désormais vers des formules comme l'intéressement à la productivité. La question du rendement ouvrier devient alors celle de la participation des salariés à une économie «rationnelle» et «concertée»; la mobilisation des salariés et leur adhésion aux programmes des entreprises sont assurées non pas par l'incitation individuelle mais par l'implication dans les collectifs de travail (MotTez, 1966). La tentative de faire converger les intérêts des entreprises avec ceux des salariés permet de soulever les questions fondamentales de l'institution salariale, questions qu'une société hantée alors

(*) Centre Maurice Halbwachs (CNRS-EHESS-ENS), équipe Pro / Laboratoire interdisciplinaire pour la sociologie économique (CNRS-Cnam); ferricciardi@yahoo.fr par le conflit social cherche à résoudre (STEINHOUSE, 2006) : comment obtenir l'adhésion du travailleur à l'ordre productif? Comment s'assurer d'une pleine participation à l'œuvre commune? Comment stabiliser les relations entre capital et travail?

Dans le sillage de ces questionnements, nous nous intéresserons à la technique de job evaluation (salaire au poste ou cotation au poste) ${ }^{(1)}$ dans le contexte des premières tentatives d'intégration européenne, à savoir la construction du marché commun du charbon et de l'acier sous l'égide de la CECA dans les années 1950 et $1960^{(2)}$. Expérimentée et développée en Amérique du Nord durant la seconde guerre mondiale, en particulier dans les industries métallurgiques, la job evaluation fait l'objet d'un certain engouement de la part non seulement de cabinets-conseil et cadres d'entreprises, mais aussi des technocrates de la CECA. Ils $\mathrm{y}$ voient un instrument pour mener à bien leur projet de régulation sociale sur fond de rationalisation de la main-d'œuvre et de stabilisation des relations professionnelles, conditions préalables à l'harmonisation d'un marché unifié.

Cette méthode vise à décrire le contenu du travail de chaque poste pour en établir la valeur relative sur une échelle de rétributions, avec pour objectif principal d'éliminer les inégalités salariales suivant le

(1) Nous utilisons indifféremment ces trois définitions plutôt que celle forgée à l'époque pour le public francophone - «qualification du travail» - , du fait qu'elle renvoie génériquement au savoir-faire des ouvriers.

(2) Les membres fondateurs de la Communauté européenne du charbon et de l'acier (CECA) étaient l'Allemagne de l'Ouest, la Belgique, la France, l'Italie, le Luxembourg et les Pays-Bas. 
principe equal pay for similar work, à l'intérieur d'un établissement comme d'une branche industrielle. C'est surtout la possibilité de relier le salaire aux caractéristiques du poste occupé, et non à la production fournie par les salariés ou à leurs savoir-faire et compétences, qui séduit les experts européens. La méthode doit permettre de rationaliser la gestion des salariés par le recours à des dispositifs de sélection à l'entrée dans le poste et de mobilité interne (ou externe), censés régler l'évolution des carrières, d'où le double qualificatif de "scientificité» et d' "équité» associé à cet outil de gestion (3) (QuAID, 1993).

Certes, derrière l'utopie scientiste cherchant à obtenir une adéquation quasi parfaite entre les moyens humains et les moyens techniques, il y a souvent des approximations, des problèmes de réglage ou des conflits ${ }^{(4)}$. En 1973, Mireille DADOY dénonce l'opposition entre, d'une part, le discours patronal et celui des experts en organisation qui mettent l'accent sur la rigueur scientifique, l'équité de la méthode, sa contribution au progrès et à la paix sociale et, d'autre part, le sentiment du personnel ouvrier, déboussolé par cet effort de rationalisation qui a pour but de faciliter leur intégration à l'entreprise (DADOY, 1973). La visée rationalisatrice n'est pas toujours atteinte en pratique. Il importe donc de déconstruire cet outil de gestion pour saisir les multiples enjeux qui y sont associés, et qui émergent notamment lors des négociations collectives (Pezet, 2000). Ainsi, il s'agira d'interroger le projet de régulation technocratique sous-jacent à la promotion de la job evaluation dans l'Europe de la CECA, en mettant l'accent sur le lien entre l'opérationnalité technique de cet outil et son inscription dans le contexte idéologique des réflexions sur l'organisation économique caractérisant l'«esprit du capitalisme» de l'époque (Boltanski, Chiapello, 1999).

\section{La CECA ou les technocrates de la régulation sociale}

Parmi les objectifs affichés par la Haute autorité du charbon et de l'acier (l'organe exécutif de la CECA) depuis sa naissance, en 1951, le volet social figure

(3) L'argument de scientificité et d'équité se retrouve également dans le cas des tentatives d'introduction du salaire au mérite dans la fonction publique, où sa mise en œuvre pose toutefois de tout autres problèmes (JoIN-LAMBERT, LOCHARD, 2010).

(4) Dans l'industrie automobile, par exemple, les mécontentements concernent aussi bien les contremaîtres (qui perdent leur pouvoir sur la définition des tâches) que les ouvriers (dont les revendications sont plus difficiles à faire aboutir à cause de la multiplication des échelons hiérarchiques) (Moutet, 2005). en bonne place ${ }^{(5)}$. Dans un esprit pionnier cherchant à asseoir les conditions d'une économie administrée à l'échelle supranationale, cette première institution "communautaire» s'engage à contribuer également à l'amélioration des conditions de vie et de travail des salariés du secteur sidérurgique et minier, dans la mesure où la protection sociale est associée au libéralisme du programme du marché commun. En effet, la régulation des problèmes de santé et de sécurité dans la sidérurgie européenne est censée assurer tout à la fois la liberté de mouvement des travailleurs et l'égalité des conditions de concurrence des entreprises. En reprenant les préconisations du Bureau international du travail (BIT) en matière de mobilité de la main-d'œuvre (ROSENTAL, 2006), les visées plus proprement sociales de la CECA sont déclinées suivant les recommandations du programme de modernisation du secteur, en termes aussi bien technologiques que de fonctionnement du marché du travail : les premières négociations des pays membres se concentrent par exemple sur le droit de migrer des travailleurs (Mechi, 2004). L'introduction du salaire au poste, c'est-à-dire d'une «convention d'équivalence générale» entre les emplois sidérurgiques, serait ainsi opérationnelle pour comparer les coûts et la productivité, ce qui permettrait également d'envisager des programmes de restructuration industrielle et de circulation intra-européenne de la main-d'œuvre (Mioche, 2004). C'est pourquoi dans l'agenda de la CECA, les problèmes de politique économique tels que l'intégration commerciale des partenaires européens, vont de pair avec les questions plus strictement techniques, comme l'accroissement de la production et de la productivité, et sociales, comme les projets de reconversion industrielle, la diffusion de la médicine du travail ou la construction de logements ouvriers (CECA, 1963).

L'intérêt de la Haute autorité pour la job evaluation témoigne de l'ensemble de ces préoccupations. Il s'agit en effet de comprendre dans quelle mesure l'utilisation de cette méthode peut contribuer à apporter des solutions aux problèmes rencontrés par «l'Homme au travail» et à accroître la productivité dans les entreprises ${ }^{(6)}$. Dès la fin de l'année 1953, plusieurs experts et fonctionnaires sont ainsi sollicités pour s'emparer de la question. Chef de la Division des problèmes du travail de

(5) Pour réaliser ce travail, les archives de la Haute autorité de la Communauté européenne du charbon et de l'acier, rattachée à la Commission européenne, ont été largement utilisées. Elles sont conservées aux Archives historiques de l'Union européenne de Florence (référence : AHUE BAC). Elles illustrent l'activité de ses différentes directions, services d'expertise et groupes de travail. Nous avons plus particulièrement consulté la documentation du groupe de travail sur la «qualification du travail», qui consiste essentiellement en des rapports d'étude, des comptes rendus de réunions et conférences, et des correspondances.

(6) AHUE BAC 1/1970-53, Étude de la qualification du travail, 20 novembre 1953. 
la CECA, service spécialisé non seulement dans les problèmes de santé des travailleurs mais aussi dans la «réadaptation et la sécurité sociale» de ceux-ci ${ }^{(7)}$, Giuseppe Glisenti est un élément moteur de cette initiative. Intellectuel provenant des cercles réformateurs du catholicisme social italien, Glisenti fait une brillante carrière de manager-négociateur au sein du groupe d'entreprises nationalisées IRI (Istituto per la ricostruzione industriale(8)), puis arrive à la tête de leur association patronale constituée en 1958 afin de mener une politique syndicale autonome de celle des entreprises privées. Aux débuts de son activité professionnelle, il dirige entre 1947 et 1951 la revue Cronache sociali ${ }^{(9)}$, foyer du débat socioéconomique alimenté par les «catholiques de gauche» italiens. Il travaille au bureau d'études de l'organisation syndicale d'inspiration chrétienne - la Confederazione italiana sindacato lavoratori $^{(10)}$, CISL - qui le propulse à la tête de la délégation italienne engagée dans les négociations pour l'institution de la CECA. Glisenti s'intéresse alors aux problématiques de coopération entre partenaires sociaux (FeLisinI, 2013, pp. 235-237). À Luxembourg, il réunit autour de lui un premier groupe d'experts venus d'horizons divers, qui est progressivement élargi et rassemble des fonctionnaires (de la Haute autorité et du BIT), des syndicalistes (de l'IG Metall) ${ }^{(11)}$ et des représentants du patronat provenant des principales entreprises sidérurgiques de l'époque comme les Aciéries de Longwy ou la société belge Cockerill(12).

L'approche qu'adopte Glisenti sur la question de la job evaluation est d'abord marquée par une certaine prudence : il s'agit moins de trancher la question salariale - qui, d'ailleurs, ne figure pas dans les missions du traité de Paris instituant la CECA que d'étudier une méthode fondée sur une analyse précise du travail et permettant de dégager des informations utiles à la formation, au recrutement et à la promotion des salariés ${ }^{(13)}$. Ainsi, lors de la plupart des rencontres qui y sont consacrées, leur caractère empirique et facultatif est rappelé alors que certains

(7) Dès le début, la CECA se charge du suivi des travailleurs impliqués dans les opérations de restructuration et de modernisation de la branche sidérurgique des pays adhérents au traité, en assurant des formes diverses de financement (indemnités chômage, aides à la requalification professionnelle, aides à la mobilité territoriale, etc.) afin de pallier les effets négatifs de la «modernisation» du secteur et accompagner la transition vers le marché commun.

(8) Institut pour la reconstruction industrielle (NDLR : toutes les traductions ont été réalisées par l'auteur de l'article).

(9) Chroniques sociales.

(10) Confédération italienne du syndicat des travailleurs.

(11) Il s'agit du grand syndicat professionnel allemand qui représente les ouvriers de l'industrie métallurgique, du textile et de l'habillement, du bois et du plastique.

(12) AHUE BAC 1/1970-53, Etude de la qualification du travail, 20 novembre 1953.

(13) AHUE BAC 1/1970-53, Qualification du travail (job evaluation), Réunion des 4 et 5 décembre à Düsseldorf, 31 décembre 1953. experts voudraient mettre en avant la supériorité de cette technique d'analyse et d'évaluation du travail, en s'appuyant sur les enseignements tirés des pays où elle a été largement appliquée comme les pays anglosaxons ${ }^{(14)}$. Cependant, on peut lire, en filigrane, la volonté des instances communautaires de faire du salaire au poste le fondement de la modernisation des politiques du travail et des relations sociales dans les entreprises, en réactualisant les connaissances du BIT en matière de classifications professionnelles ${ }^{(15)}$. Se dessine ainsi un rapport de collaboration entre les experts du BIT et de la CECA (appartenant, parfois, aux mêmes réseaux de réformateurs d'inspiration chrétienne) autour des problématiques de rationalisation du travail et de la protection sociale.

C'est ce dont témoigne une lettre adressée par Jean-Pierre Després, fonctionnaire du BIT, ancien directeur du Département des relations industrielles à l'Université de Laval au Québec et issu des cercles du catholicisme social québécois, à son collègue de la Haute autorité, Charles Savouillan, ancien secrétaire national de la fédération métallurgique de la CFTC (16), concernant le projet d'enquête sur la job evaluation mis en chantier par la Haute autorité en 1953. Il y est surtout question de choix sémantiques, dans la mesure où il faudrait distinguer entre les termes de job analysis (l'étude scientifique de tous les faits ou éléments qui se rapportent à une tâche), job description (l'étude des profils d'ouvriers possédant les aptitudes requises pour effectuer les tâches) et job evaluation (l'étude de l'importance relative des tâches). Le projet d'enquête de la Haute autorité semble se concentrer davantage sur la job description, et offre donc l'opportunité de saisir la réalité sociale de l'usine dans son ensemble :

«Par le biais de cette étude sur la qualification du travail [...] vous aurez l'occasion d'examiner plusieurs questions qui relèvent de la direction du personnel, notamment le recrutement, la formation, les transferts, l'avancement, etc. Indirectement vous pourrez vous rendre compte des causes du malaise qui peut exister à l'intérieur d'une usine donnée ou plus exactement des causes des griefs, fondés ou non, des ouvriers à l'égard de l'organisation scientifique du travail dans une entreprise déterminée. En outre, vous pourrez recueillir des indications utiles en matière de mouvement de la main-d'œuvre et de sécurité de travail. En d'autres termes, $[\ldots]$ vous obtiendrez des bases qui permettront d'étudier plus tard les divers problèmes qui relèvent

(14) Pour des premiers éléments de ce débat, $c f$. AHUE BAC $1 / 1970-52$, Étude «job evaluation» dans l'industrie sidérurgique, 7 mai 1956.

(15) En 1950, à l'occasion de la Conférence internationale sur la qualification du travail, organisée à Genève sous les auspices du Comité international de l'Organisation scientifique du travail, la job evaluation avait fait l'objet d'un rapport d'étude. D'autres études sont réalisées successivement, par exemple par la sous-commission des méthodes d'évaluation du travail dans les industries mécaniques, instituée lors de la sixième session de la Commission des industries mécaniques de l'Organisation internationale du travail en 1957 (BIT, 1960).

(16) Confédération française des travailleurs chrétiens. 
du domaine de la psychosociologie industrielle et des relations professionnelles en général. »(17)

Loin de se résumer à la résolution de simples problèmes techniques, la méthode de la cotation au poste serait en mesure de fournir les informations indispensables à une gestion plus rationnelle et efficace de la main-d'œuvre, en contribuant également à stabiliser les relations sociales dans les entreprises.

$\mathrm{Au}$ fondement de ce raisonnement se trouvent deux constats : l'application de méthodes d'évaluation du travail s'expliquerait par la «poussée des organisateurs désirant remplacer des bases de classifications arbitraires et sans fondement par d'autres déterminées scientifiquement» ainsi que par les nombreuses revendications qui émergent dans les usines autour de la question des hiérarchies professionnelles ${ }^{(18)}$. Les techniques modernes ayant fait "éclater» les métiers traditionnels, «les règles qui permettent la classification des postes de travail dans la hiérarchie professionnelle, ainsi que les barèmes de salaires qu'elles déterminent, [seraient] insuffisants, étant inadaptés aux problèmes posés par les techniques modernes de production et d'organisation du travail»(19). Autrement dit, le progrès technique que connaît alors le secteur sidérurgique (notamment dans la phase de laminage qui, de manuelle, devient automatisée) remet en cause les accords nationaux et de branche sur les grilles professionnelles, désormais critiqués par les représentants des organisations professionnelles, notamment patronales ${ }^{(20)}$. Ces accords sont en effet restés les mêmes depuis vingt ou trente ans, tant sur les principes que sur la forme, et ont conduit à des ajustements ex-post susceptibles de créer des situations de conflit(21). Les paroles de Savouillan expriment bien cette approche «révisionniste» :

«On fait davantage appel à des aptitudes par exemple de tension nerveuse. C'est une forme d'aptitude, de responsabilité. L'ouvrier doit faire davantage attention parce qu'il travaille sur une machine qui coûte très cher alors que, précédemment, on faisait davantage appel à de la formation professionnelle en ce qu'il (sic) concerne le travail qu'il avait à exécuter... tandis que maintenant on lui demande d'autres aptitudes que celles nées strictement de la formation professionnelle.»(22)

(17) AHUE BAC 1/1970-51, Lettre de Després à Savouillan, 14 novembre 1953 .

(18) AHUE BAC 1/1970-53, Étude de la qualification du travail, 20 novembre 1953.

(19) AHUE BAC 1/1970-52, Les accords de salaire et la job evaluation, pour les ouvriers sidérurgiques de la CECA, 19 janvier 1956.

(20) AHUE BAC 237/1980-26, Journées d'étude sur la qualification du travail (job evaluation) dans la sidérurgie de la Communauté, 9 décembre 1958.

(21) AHUE BAC 1/1972-52, Les accords de salaire et la job evaluation, pour les ouvriers sidérurgiques de la CECA, 19 janvier 1956.

(22) AHUE BAC 1/1970-52, Étude «job evaluation» dans l'industrie sidérurgique, 7 mai 1956, p. 5.
Cette conception s'appuie sur une analyse évolutionniste du changement technique considéré comme capable de déterminer de manière unilatérale l'organisation du travail et les relations de travail. Cette vision est à l'époque partagée, bien qu'à partir de points de vue divergents, par des cadres dirigeants, des syndicalistes et aussi par de nombreux chercheurs en sciences sociales. Les enquêtes menées par plusieurs équipes de sociologues sous l'égide (et grâce au financement) de la CECA témoignent de cette tendance : les transformations de l'organisation du travail sont ainsi rythmées par les changements techniques, indépendamment du contexte social de production. Ces études se focalisent sur le rapport entre niveau de mécanisation et implication des salariés, et sur l'évolution conséquente des modes de rémunération (LuTZ, WiLlener, 1960). L'accent est donc mis sur le processus de «décomposition du travail»-pour reprendre l'expression d'Alain Touraine, l'un des animateurs de ces enquêtes - notamment lorsque l'analyse vise à saisir de plus près la nature des pratiques de travail (Dofny et al., 1966; DuRAND et al., 1972) ${ }^{(23)}$. Se justifie par conséquent le recours à de nouvelles méthodes d'évaluation des postes qui permettent de dépasser le décalage entre les «textes à appliquer» (les hiérarchies professionnelles) et la «réalité industrielle»(24).

\section{Une comparaison à l'échelle européenne : entre accords collectifs et hiérarchies professionnelles}

La Haute autorité n'entend pas faire ouvertement de propagande en faveur de la job evaluation. Il n'en reste pas moins que l'attitude de ses porteparoles penche décidément vers la révision des anciens accords de salaire, adossés aux grilles de catégories professionnelles. Après avoir publié, en 1957, des monographies nationales sur l'application de la cotation au poste (environ $20 \%$ des ouvriers sidérurgistes de la Communauté sont concernés) (25), l'occasion de faire le point sur «l'état de l'art» se présente lors de l'organisation,

(23) Pour une critique plus détaillée de ces enquêtes, voir LOMBA (2001).

(24) AHUE BAC 237/1980-26, Journées d'étude sur la qualification du travail (job evaluation) dans la sidérurgie de la Communauté, 9 décembre 1958.

(25) Si l'on ne tient pas compte de l'Allemagne, en 1958 seulement $10 \%$ des entreprises de la CECA (sur la base du nombre d'ouvriers en poste) sont touchés par la méthode de cotation au poste. Cf. AHUE BAC 237/1980-27, CECA-Haute autorité, Rapport 3, Développement de la Job Evaluation, Règles contractuelles et Job Evaluation, Processus d'application dans les exemples choisis, 10-12 décembre 1958, p. 2. Parmi ces monographies, nous avons notamment consulté celle concernant l'Italie (CECA, 1957). 
en décembre 1958, d'une session d'étude consacrée à la synthèse des travaux menés jusque-là qui permet également une confrontation des opinions des milieux professionnels ${ }^{(26)}$. Or il s'avère que la méthode de cotation au poste ne fait pas l'unanimité, pas même chez ses partisans, qui sont divisés sur l'établissement d'une échelle des salaires, l'intégration des primes au rendement ou encore, le rôle des conventions collectives ${ }^{(27)}$. Ses adversaires, quant à eux, considèrent cette méthode comme «trop rigide, trop abstraite, trop détachée de la réalité du marché du travail et de ses fluctuations, trop limitative de l'action des organisations professionnelles, etc. ${ }^{(28)}$.

Tableau 1 : Critères habituellement retenus par pays pour l'évaluation du travail manuel d'après la méthode de job evaluation (1958)

\begin{tabular}{|c|c|c|c|c|}
\hline Belgique & France & Italie & Pays-Bas & Allemagne occidentale \\
\hline Intelligence & $\begin{array}{l}\text { Apprentissage } \\
\text { ou formation } \\
\text { professionnelle } \\
\text { Expérience } \\
\text { professionnelle }\end{array}$ & $\begin{array}{l}\text { Exigences } \\
\text { intellectuelles } \\
\text { Période de formation et } \\
\text { d'expérience nécessaire } \\
\text { Capacités et } \\
\text { dispositions } \\
\text { intellectuelles }\end{array}$ & $\begin{array}{l}\text { Réglage et conduite de machines } \\
\text { Travaux exécutés d'après dessin } \\
\text { Travaux effectués avec } \\
\text { instruments et appareils de } \\
\text { mesure } \\
\text { Appréciation et choix de } \\
\text { matériaux } \\
\text { Travaux de nature spéciale }\end{array}$ & $\begin{array}{l}\text { Connaissance } \\
\text { professionnelle = } \\
\text { formation professionnelle } \\
+ \text { expérience } \\
\text { professionnelle }\end{array}$ \\
\hline Habileté & --- & Habileté manuelle & $\begin{array}{l}\text { Habileté } \\
\text { Précision dans l'exécution du } \\
\text { travail } \\
\text { Petites pièces à usiner }\end{array}$ & Habileté professionnelle \\
\hline Responsabilité & $\begin{array}{l}\text { Responsabilité en ce qui } \\
\text { concerne : } \\
\text { - le matériel de } \\
\text { l'entreprise } \\
\text { - les pièces à usiner } \\
\text { - la sécurité d'autrui } \\
\text { - le déroulement du } \\
\text { travail }\end{array}$ & $\begin{array}{l}\text { Responsabilité en ce qui } \\
\text { concerne : } \\
\text { - l'outillage, les } \\
\text { appareils et les } \\
\text { machines } \\
\text { - les matières } \\
\text { - la sécurité d'autrui } \\
\text { - le déroulement du } \\
\text { travail }\end{array}$ & $\begin{array}{l}\text { Risques de détérioration des } \\
\text { machines et installations } \\
\text { Risques de détérioration de } \\
\text { l'outillage et des pièces à usiner } \\
\text { Responsabilité vis-à-vis d'autrui } \\
\text { Responsabilité dans le cadre du } \\
\text { travail d'équipe }\end{array}$ & $\begin{array}{l}\text { Responsabilité en ce qui } \\
\text { concerne : } \\
\text { - le matériel de l'entreprise } \\
\text { et les produits fabriqués } \\
\text { - la sécurité d'autrui } \\
\text { - le déroulement du travail }\end{array}$ \\
\hline $\begin{array}{l}\text { Efforts } \\
\text { Position du corps }\end{array}$ & Efforts physiques & $\begin{array}{l}\text { Efforts physiques } \\
\text { exigés }\end{array}$ & $\begin{array}{l}\text { Position du corps } \\
\text { Port d'objets lourds }\end{array}$ & $\begin{array}{l}\text { Efforts musculaires } \\
=\text { difficulté du travail } \\
+ \text { capacité de travail } \\
\text { (constitution) }\end{array}$ \\
\hline $\begin{array}{l}\text { Capacité visuelle } \\
\text { Capacité auditive }\end{array}$ & Efforts intellectuels & $\begin{array}{l}\text { Efforts intellectuels et } \\
\text { visuels }\end{array}$ & --- & $\begin{array}{l}\text { Attention } \\
\text { Réflexion }\end{array}$ \\
\hline $\begin{array}{l}\text { Conditions visuelles } \\
\text { Conditions acoustiques } \\
\text { Atmosphère chaude ou } \\
\text { froide } \\
\text { Atmosphère insalubre }\end{array}$ & Conditions de travail & Environnement & $\begin{array}{l}\text { Conditions atmosphériques sur } \\
\text { le poste de travail }\end{array}$ & $\begin{array}{l}\text { Environnement } \\
\text { Température } \\
\text { Eau, acides } \\
\text { Salissures, poussières } \\
\text { Gaz, vapeurs, humidité } \\
\text { Bruits, vibrations } \\
\text { Éblouissement, manque de } \\
\text { lumière } \\
\text { Risque de refroidissement }\end{array}$ \\
\hline--- & Accidents & Risques & Risques pour la santé & Risques d'accident \\
\hline Commandement & $\begin{array}{l}\text { Risques de } \\
\text { déclassement en fin de } \\
\text { carrière }\end{array}$ & --- & $\begin{array}{l}\text { Nature du travail } \\
\text { Durée du cycle de travail } \\
\text { Diversité et complexité des } \\
\text { pièces à usiner } \\
\text { Conduite de plusieurs machines } \\
\text { ou installations }\end{array}$ & 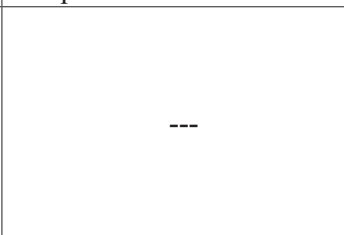 \\
\hline
\end{tabular}

Source : AUE BAC 237/1980-27, CECA-Haute autorité, Rapport 2, La Job Evaluation : but, notions de base, méthodes en vigueur (10-12 décembre 1958), pp. 28-29.

(26) Participent à cette session d'étude, qui a lieu du 10 au 12 décembre, environ 140 représentants des organisations syndicales et patronales, ainsi que des membres de la Commission des affaires sociales de l'Assemblée parlementaire européenne, de la Commission économique européenne, du Bureau international du travail et de l'Agence européenne de productivité.
(27) AHUE BAC 237/1980-27, Note d'information pour MM. les membres de la Haute autorité, 16 décembre 1958. (28) Ibid. 
L'analyse approfondie des rapports précédemment évoqués permet de dégager quelques lignes de force. De prime abord, le sentiment qui prévaut à leur lecture est celui d'une grande incertitude quant aux mises en œuvre de la job evaluation : les différents contributeurs s'empressent en effet de souligner l'hétérogénéité et la spécificité des solutions apportées dans chaque pays, rendant toutes comparaisons à l'échelle européenne sinon impossibles, du moins laborieuses. Ces comparaisons sont alors conduites sur les notions de base que suppose la mise en œuvre de la méthode dans les différents pays de la Communauté. Si l'on regarde les critères d'évaluation sur lesquels porte l'application du salaire au poste, les différences sont nombreuses. En France, par exemple, on estime que l'habileté manuelle ne doit pas être prise en compte car son appréciation peut paraître trop subjective. En outre, l'importance qu' on attribue à chaque critère varie d'un pays à l'autre de sorte que l'évaluation chiffrée donne lieu à des résultats contrastés ${ }^{(29)}$. Étant donné que les définitions des différents critères d'évaluation ne concordent pas, le tableau synoptique censé les présenter ne permet aucune réelle comparaison (voir tableau 1). Il se contente de donner une vue d'ensemble. Certes, il y a une relative convergence autour des grandes catégories de critères à retenir pour l'évaluation (formation et expérience professionnelle, responsabilité, effort physique, environnement de travail, etc.), mais leur contenu peut parfois différer de manière sensible.

Comment, malgré tout, comparer des situations si diverses? Une solution consiste à faire varier la focale en prenant comme point d'entrée la situation antérieure dans les pays considérés, c'est-à-dire en mesurant les résultats de l'application de la job evaluation par rapport aux anciennes grilles professionnelles issues des conventions collectives nationales ou des accords locaux. C'est ce que suggère le rapport de synthèse $n^{\circ} 4$, rédigé par Léopold Dor, docteur en mathématiques, chef du Service d'organisation de la société belge CockerillOugrée, et considéré comme l'un des principaux experts européens sur le sujet(30). Si la démarche entreprise diffère d'une nation à l'autre, le but poursuivi semble être similaire, à savoir réviser les systèmes de catégories professionnelles (généralement jugées dépassées) tout en essayant d'intégrer les nouvelles techniques d'évaluation du travail dans les accords collectifs existants. Plutôt que d'un

(29) AHUE BAC 237/1980-27, CECA-Haute autorité, Rapport 2, La Job Evaluation : but, notions de base, méthodes en vigueur, 10-12 décembre 1958.

(30) AHUE BAC 237/1980-27, CECA-Haute autorité, Rapport 4, Les résultats de l'application de la qualification du Travail, Comparaison avec la situation antérieure, 10-12 décembre 1958. Propagateur des méthodes mathématiques de classification dans l'entreprise, Dor utilise son expertise pour promouvoir sa carrière à la fois chez Cockerill (il en devient le chef du personnel) et dans le monde académique (il enseigne les statistiques à l'Université de Liège) (LOMBA, 2004). changement radical, il s'agit donc d'une adaptation, qui donne lieu à des recompositions ou à des superpositions entre les hiérarchies traditionnelles et les hiérarchies issues de la cotation au poste.

L'exemple allemand est, à cet égard, éclairant. Si la plupart des ouvriers sidérurgistes allemands sont concernés par la méthode d'évaluation du travail (on estime qu'en 1955 environ $44 \%$ des salariés du secteur y sont soumis), celle-ci n'est cependant pas intégrée dans les accords collectifs de portée régionale et figure uniquement dans les accords d'entreprise. Le système traditionnel des catégories professionnelles, qui varie suivant les régions ${ }^{(31)}$, demeure ainsi le référentiel de base pour la hiérarchisation du personnel, d'autant que le syndicat IG Metall semble s'y accrocher afin de garder la main sur le contrôle du salaire au rendement. En effet, «l'évaluation du travail n'avait rien à voir avec le montant, les variations et les bases de calcul de la part du gain représentant le salaire au rendement»(32). Ceci explique pourquoi l'attitude des syndicalistes allemands face à cette nouvelle méthode reste prudente, et seulement modérément positive : ils sont convaincus que son application ne permet pas de résoudre tous les problèmes, notamment celui de la juste répartition du salaire entre salaire fixe et salaire variable ${ }^{(33)}$. Son acceptation passe donc par la règle «salaire normal pour rendement normal» : toute part du salaire provenant d'un rendement supplémentaire (bonus de rendement, primes, etc.) ne doit pas relever, pour ces syndicalistes, de la job evaluation mais de l'évaluation du rendement. Sont ensuite définis un certain nombre de "principes syndicaux relatifs à l'évaluation des tâches", qui posent les conditions préalables à la mise en œuvre du salaire au poste en termes de co-gestion de celui-ci : intangibilité des clauses normatives et matérielles des conventions collectives, institution de commissions paritaires d'évaluation des postes, refus de toute forme de travail et de rémunération introduites par une initiative unilatérale des employeurs, refus de toute diminution des salaires ouvriers, etc. ${ }^{(34)}$

(31) En Rhénanie du Nord-Westphalie, par exemple, la classification professionnelle s'articule en cinq catégories (manœuvre, ouvrier spécialisé, ouvrier qualifié, ouvrier spécialisé des services de production sidérurgique, ouvrier hautement qualifié); elle est par la suite modifiée par de nouvelles conventions collectives tout au long des années 1950.

(32) AHUE BAC 237/1980-27, CECA-Haute autorité, Rapport 4, Les résultats de l'application de la qualification du Travail, Comparaison avec la situation antérieure, 10-12 décembre 1958 , p. 5.

(33) Le risque majeur est celui d'imputer à la partie du salaire au rendement les éléments de différenciation du travail (notamment inhérents aux conditions de travail et à l'effort demandé) qui auraient pu être considérés dans le cadre de l'évaluation objective des tâches. $C f$. IRI (1962), p. 66.

(34) AHUE BAC 237/1980-27, CECA-Haute autorité, Rapport 7, Attitudes devant la Job Evaluation, 10-12 décembre 1958, pp. 1-3. 
Les autres pays de la Communauté ont aussi leurs spécificités. En Belgique, faute d'accords nationaux sur les classifications professionnelles, ce sont les entreprises et les syndicats du bassin liégeois qui s'intéressent à la question de l'harmonisation des salaires dans un esprit strictement paritaire puisque c'est une commission technique paritaire qui préside, à l'échelon de la région, les travaux d'application de la cotation au poste. Aux Pays-Bas, la démarche est dirigiste : toutes les modifications apportées à des conventions collectives - y compris celles relatives à la convention spéciale axée sur la job evaluation adoptée en 1953 - doivent être approuvées par le Collège des contentieux qui est un organisme gouvernemental. En France, persistent les classifications des emplois de branche, dérivation des grilles Parodi établies en 1945, l'évaluation «objective» du travail étant déjà connue par le biais de la méthode Bedaux d'analyse des tâches (Moutet, 1997). En Italie, les catégories professionnelles traditionnelles en vigueur dans l'industrie métallurgique ne sont pas remises en cause; l'usine de Cornigliano fait néanmoins figure d'exception car elle ouvre la voie à l'application de la cotation au poste grâce à un accord collectif local qui traduit, en les réactualisant, ses besoins en matière de grilles d'emplois pour la sidérurgie ${ }^{(35)}$.

Bref, jouent de tout leur poids, d'une part, la tradition des accords collectifs opérant à plusieurs échelles et, d'autre part, les classifications de catégories professionnelles déjà existantes. Cette double dépendance contribue à brouiller les cartes, dans la mesure où toute classification ne relève pas d'un dispositif d'appréciation cristallisé et immuable, mais dépend d'un spectre plus large d'éléments. Malgré le caractère cartésien et analytique de la cotation au poste, force est de constater que la relativité des critères adoptés lors de son application résulte d'une série de conventions et de jugements de valeur solidement ancrés dans la tradition et l'histoire sociale et syndicale de chaque pays. Pour le dire avec les mots de l'expert belge Dor, il importe de souligner que :

«la qualification du travail reste en grande partie conventionnelle et que, de ce fait, sa valeur finale sera fonction de l'accord général admis au départ. Cette conclusion générale est d'autant plus importante que la tradition et/ou les institutions sont puissantes, c'està-dire qu'il est difficile de sortir des sentiers battus. La nature des résultats dans les pays de la Communauté est, constatons-le, très liée localement à l'ancienneté

(35) AHUE BAC 237/1980-27, CECA-Haute autorité, Rapport 4, Les résultats de l'application de la qualification du Travail, Comparaison avec la situation antérieure, 10-12 décembre 1958. Sur les règles contractuelles développées pour l'application du salaire au poste, voir aussi : CECA-Haute autorité, Rapport 3, Développement de la Job Evaluation, Règles contractuelles et Job Evaluation, Processus d'application dans les exemples choisis, 10-12 décembre 1958. de la sidérurgie et au pouvoir de discussion des syndicats $\gg(36)$.

La dimension contingente et «localisée» des déterminants de l'application du salaire au poste dans chaque pays semble déranger quelque peu les technocrates de la CECA, comme en témoignent certaines questions soulevées par le rapport rédigé par Dor qui recherchent une vérité contrefactuelle difficile à démontrer :

«Si la politique nationale des Pays-Bas n'avait pas été dirigiste, les usines sidérurgiques auraient-elles appliqué la J[ob] E[valuation]? [...]

Si la Société Cornigliano n'avait pas été une usine entièrement nouvelle, aurait-elle pensé à imposer la $\mathrm{J}[\mathrm{ob}] \mathrm{E}$ [valuation] ? [...]

Si la poussée salariale à Liège n'avait pas été désordonnée ${ }^{(37)}$, l'harmonisation inter-usines aurait-elle eu lieu? $[\ldots] \gg(38)$.

\section{La job evaluation à l'épreuve de la négociation collective : l'exemple de l'usine de Cornigliano (Italie)}

Dans les documents de la Haute autorité que nous avons examinés, l'expérience de l'usine italienne de Cornigliano, près de Gênes, est évoquée à plusieurs reprises en raison de son exemplarité. Le fait que l'usine ait été créée ex nihilo, et que ses dirigeants aient pu introduire le principe de la job evaluation en passant par une analyse systématique de tous les emplois, font de Cornigliano une véritable expérimentation in vitro à étudier de plus près ${ }^{(39)}$. Surtout, l'analyse et l'évaluation du travail y sont considérées comme un moyen et non comme une fin, «un moyen d'arriver à une organisation plus rationnelle du travail, un moyen permettant de résoudre divers problèmes humains et techniques » : cette double dimension technique et sociale de l'outil de gestion

(36) AHUE BAC 237/1980-27, CECA-Haute autorité, Rapport 4, Les résultats de l'application de la qualification du Travail, Comparaison avec la situation antérieure, 10-12 décembre 1958, p. 20.

(37) Il s'agit de la montée des revendications salariales à l'échelle de la branche.

(38) AHUE BAC 237/1980-27, Journées d'étude sur la Job Evaluation, Questions relatives au Rapport 4 (L. DOR), n. d. [1958].

(39) L'usine de Cornigliano, construite au début des années 1950 grâce aux financements de l'État italien et du plan Marshall, est au cœur du projet de modernisation industrielle porté par les pouvoirs publics, qui passe notamment par la capacité de se doter d'un secteur sidérurgique compétitif, capable de répondre aux besoins de toute la filière métallurgique et s'appuyant sur une technologie moderne (le train de laminage continu). Plus précisément, il s'agit d'un établissement consacré à la fabrication de tôles plates dont la plus grande partie est destinée à l'industrie automobile et électroménagère. En 1960, sa production dépasse le seuil d'un million de tonnes d'acier, à peu près $20 \%$ du total de la production à l'échelle nationale. 
retient l'attention des dirigeants de l'usine(40). Autrement dit, le salaire au poste présuppose une convergence vertueuse entre politiques syndicales et organisation du travail afin de pouvoir obtenir une meilleure intégration des salariés ainsi qu'une plus grande responsabilisation des organisations syndicales ${ }^{(41)}$.

La méthode adoptée est celle de la société américaine $\mathrm{ARMCO}^{(42)}$, dont certains cadres font le déplacement à Cornigliano pour en assurer l'expertise initiale, qui permettra ensuite sa mise en œuvre. Il s'agit d'une procédure articulée en plusieurs étapes - description du poste, évaluation de celui-ci sur la base de plusieurs critères, définition de la rémunération correspondante, etc. - qui débouche sur la répartition des ouvriers sur une échelle de vingt-quatre «classes de rémunérations 》 (voir encadré). Si, d'après les dirigeants de l'entreprise, la nouvelle méthode d'évaluation du travail devait assurer des rétributions plus appropriées et une utilisation plus rationnelle de la main-d'œuvre, première étape pour donner un nouvel élan à la négociation collective (FASCETTI, 1959), force est de constater qu'au début, son introduction n'a pas été discutée. Les conditions «exceptionnelles» caractérisant la phase de construction de l'établissement - pénurie de main-d'œuvre, difficultés techniques dans l'achèvement du chantier, etc. - expliquent au moins en partie le choix de la direction d'adopter une démarche unilatérale de mise en œuvre de la politique du personnel (DORIA, 2004-2005). La plupart des ouvriers recrutés, environ 3000 dans la première moitié des années 1950, n’ont ni formation professionnelle adéquate ni affiliation syndicale avérée. Ils proviennent de l'émigration interrégionale et sont incités, de gré ou de force, à adhérer au syndicat d'inspiration chrétienne CISL, très proche de la direction. Dans un contexte local fortement ancré à gauche ${ }^{(43)}$, la nouvelle société sidérurgique penche sans hésitation vers la normalisation politique de la main-d'œuvre, en laissant aux représentants de la CISL la mainmise sur la sélection des salariés lors de l'embauche. Cette stratégie se justifie aussi à l'aune des pressions politiques nationales et internationales qui sont exercées sur le site de Cornigliano où interviennent notamment hommes

(40) AHUE BAC 237/1980-27, CECA-Haute autorité, Rapport 4, Les résultats de l'application de la qualification du Travail, Comparaison avec la situation antérieure, 10-12 décembre 1958, p. 10.

(41) C'est notamment l'avis des experts du bureau d'études sur le travail opérant au sein du groupe industriel IRI, dont l'usine de Cornigliano fait partie (IRI, 1960).

(42) Il s'agit de l'American rolling mill corporation, acteur «moyen» de la sidérurgie américaine (trois millions de tonnes d'acier de production annuelle, 25000 salariés), qui assiste la Cornigliano dans l'installation et l'entretien des équipements techniques (notamment le train de laminoirs).

(43) Le Parti communiste et la centrale syndicale Confederazione generale italiana del lavoro (CGIL; [Confédération générale italienne du travail]) sont très implantés dans la ville de Gênes. politiques, évêques et diplomates américains, et qui font de celui-ci une véritable vitrine de l'affirmation d'un nouveau modèle de relations sociales de type coopératif et non conflictuel (et surtout à l'abri de l'influence communiste!) (RICCIARDI, 2010).

\section{Encadré}

\section{La méthode de classement des ouvriers à Cornigliano, 1955-1956}

Un document rédigé par les enseignants de l'école de gestion IPSOA de Turin - la première business school italienne - lors d'une visite à l'établissement de Cornigliano, décrit les modes de fonctionnement de la méthode de job evaluation qui y est appliquée.

Après avoir identifié les postes de travail nécessaires pour chaque secteur de la production, les experts du service des méthodes, formés aux ÉtatsUnis, rédigent une «fiche descriptive» contenant les informations principales du job : dénomination, tâche caractérisant le poste, machines, outillage, matériaux et produits utilisés, position hiérarchique en termes de dépendance et de responsabilité, détail des opérations à accomplir, etc. Ensuite, ils procèdent à l'évaluation du poste en s'appuyant sur cinq catégories de conditions standard (conditions intellectuelles et manuelles, conditions relatives à l'exercice de la responsabilité, effort demandé, conditions de travail, risque), lesquelles se décomposent plus finement en douze critères d'évaluation. À l'aide du manuel d'évaluation précédemment préparé, ils assignent à chacun de ces critères des valeurs (A, B, C, etc.), auxquels correspondent des points, dont la somme finale sert à déterminer la «zone technologique» relative au poste examiné. Celle-ci, enfin, est transformée en une des vingtquatre "classes de rémunération " qui sont utilisées pour déterminer les salaires. La rédaction d'une fiche descriptive demande en moyenne entre huit et dix heures par poste. Elle est effectuée par un ingénieur des méthodes qui a été formé pour cela au moins pendant six mois.

Source : IPSOA (1955-1956).

Les sources syndicales, notamment celles issues de la fédération métallurgique locale affiliée à la CGIL, nous offrent la possibilité de rendre compte autrement de la situation, en mettant en avant le regard des pourfendeurs du salaire au poste(44). L'application de cette technique passe en effet aussi par des accords collectifs signés sur place avec toutes les organisations syndicales et/ou leurs représentants. Ces compromis interrogent de manière inédite les questions de la qualification des

(44) Il s'agit, pour l'essentiel, de compte rendus de réunions, rapports d'étude, notes et accords collectifs issus de l'activité du syndicat métallurgique Federazione impiegati operai metallurgici (FIOM, affilié à la centrale CGIL), dont la plupart sont conservés au Centro ligure di storia sociale [Centre ligure d'histoire sociale] de Gênes (référence : CLSS AFIOM). 
travailleurs et de leur inscription dans l'organisation du travail.

Un premier accord entre la direction et la commission interne, qui est l'organisme des représentants des salariés, est conclu en septembre 1956. L'objectif est de supprimer la distinction, caractéristique de la sidérurgie italienne, entre les salaires au poste pour les ouvriers de fabrication et les salaires définis par la qualification pour les ouvriers de métier, employés dans les activités de service et d'entretien ${ }^{(45)}$. La décision est donc prise de classer tous les ouvriers dans le premier groupe salarial, celui des ouvriers de fabrication, en articulant plus finement la grille des emplois (tourneur, fondeur, grutier, etc.), avec les résultats de l'opération d'analyse des postes réalisée par les bureaux des méthodes. Le seul élément discriminant devient par conséquent le poste ${ }^{(46)}$.

Un autre accord est signé fin 1959 à la suite de la mobilisation spontanée de plusieurs groupes de salariés, notamment les ouvriers opérant à l'aciérie et au laminoir à chaud. Ils demandent que la part de salaire au rendement encore en vigueur, de fait bloquée sur des niveaux qui ne peuvent pas être atteints, soit révisée. En même temps, ils réclament l'extension de l'application du système de rémunération au poste pour lequel des standards de qualification obéissant aux critères psychophysiologiques de la job description ont été fixés et sont largement reconnus par les ouvriers. Le conflit débouche sur un compromis qui, d'une part, maintient la rémunération au mérite en la diminuant cependant (le seuil de la prime de rendement passe de $25 \%$ à $17 \%$ ) et, d'autre part, mise sur la stabilisation des rétributions en fonction du poste occupé (le salaire fixe augmente de 4,5\%) ${ }^{(47)}$. Cet accord s'inscrit dans un mouvement généralisé de contestation du salaire au rendement qui, à l'époque, touche le secteur sidérurgique dans toute l'Europe occidentale (LutZ, WilLENER, 1960).

Effet inattendu : cette mobilisation «par le bas » permet aux syndicats jusque-là opposés à la job evaluation - et qui ont su, comme l'a fait la CGIL, accompagner les revendications ouvrières - d'entrer dans le jeu de la négociation et d'acquérir une

(45) Dans la sidérurgie italienne, depuis les années 1940, il existe deux modes de rétribution : les salaires au poste (paghe di posto) et les salaires définis par la qualification (paghe di qualifica). Cette différenciation par la qualification se retrouve de fait également pour les ouvriers opérant dans le secteur de fabrication puisqu'il leur en est également attribué une qui intègre par la suite le périmètre d'une des quatre catégories professionnelles définies par le contrat de branche : manœuvre (manovale), ouvrier spécialisé (manovale specializzato), ouvrier qualifié (operaio qualificato) et ouvrier hautement qualifié (operaio specializzato) (GIUGNI, 1953).

(46) CLSS AFIOM b. 50, «Accordo per le paghe di classe 4/9/1956 - 7/9/1962» [Accord sur le salaire au poste].

(47) CLSS AFIOM b. 48, «Documento sulle rivendicazioni degli operai alla Cornigliano» [Document sur les revendications des ouvriers de l'établissement de Cornigliano] (1960). nouvelle légitimité dans le système de relations professionnelles. Ainsi, l'accord d'entreprise qui suit peu après, en décembre 1960, pose les bases d'une première forme d'institutionnalisation des relations professionnelles : définition conjointe du manuel fixant les critères d'analyse et de description des postes, institution de commissions mixtes pour la résolution des problèmes liés à l'application de la nouvelle méthode, engagement des syndicats à renoncer à toute forme de mobilisation ou de revendication pendant une période donnée, etc. ${ }^{(48)}$ Fruit d'un nouveau compromis, l'accord en question donne lieu, d'un point de vue technique, à une forme d'hybridation dans la définition des hiérarchies de postes, dans la mesure où réapparaissent les anciennes catégories professionnelles réaménagées, dans lesquelles sont introduites les «classes de rémunération» issues de la job evaluation (voir tableau 2).

Cette hybridation s'explique aussi par des motifs liés à la sauvegarde de l'identité ouvrière que l'intégration en 1961 de l'usine de Cornigliano dans un groupe industriel plus large et diversifié, comprenant une dizaine d'établissements, rend encore plus pressante. La présence de nombreux «ouvriers de métier», notamment d'ouvriers qualifiés et militants communistes dans les autres établissements du groupe, conduit, dans la nouvelle phase de négociation, à la révision de certains facteurs d'évaluation des postes : un poids majeur est par exemple attribué aux caractéristiques du travail sidérurgique telles que l'expérience professionnelle et la pénibilité(49). C'est ainsi que se prononcent les ouvriers de l'ILVA, ancien établissement de la province génoise fabricant des produits profilés, qui fait partie du nouveau conglomérat sidérurgique :

« tout en reconnaissant la validité du manuel d'évaluation du travail, afin d'examiner les tâches et assigner les classes de rémunération, nous retenons que ces classes ne peuvent pas remplacer les qualifications, et encore moins les catégories [professionnelles]»(50).

(48) CLSS AFIOM b. 46, «Accordo del 9 dicembre 1960» [Accord du 9 décembre 1960].

(49) CLSS AFIOM b. 45, «Punto sulle trattative tra le organizzazioni sindacali e l'Italsider per il manuale di valutazione delle paghe di classe e l'inquadramento professionale per le aziende a ciclo integrale» [État des lieux des négociations entre les organisations syndicales et la société Italsider pour la rédaction du manuel concernant la job evaluation et l'encadrement professionnel des entreprises sidérurgiques opérant à cycle intégral] (1961).

(50) Ibid., p. 4. 
Tableau 2 : Grille de classifications et de rémunérations du personnel ouvrier, usine de Cornigliano, 1961-1962*

\begin{tabular}{|c|c|c|c|c|c|c|}
\hline Groupe & $\begin{array}{c}\text { Catégorie } \\
\text { professionnelle }\end{array}$ & $\begin{array}{c}\text { Part dans } \\
\text { l'effectif total }\end{array}$ & $\begin{array}{c}\text { Classe de } \\
\text { rémunération }\end{array}$ & $\begin{array}{c}\text { Salaire/heure } \\
1961\end{array}$ & $\begin{array}{c}\text { Salaire/heure } \\
1962\end{array}$ & Différence + /- \\
\hline \multirow{3}{*}{ I } & \multirow{3}{*}{$\begin{array}{l}\text { Ouvrier } \\
\text { spécialisé } 1^{\mathrm{er}} \\
\text { degré }\end{array}$} & \multirow{3}{*}{$2,4 \%$} & Base & 215,50 & 215,50 & $=$ \\
\hline & & & 1 & 220 & 221,86 & $+1,86$ \\
\hline & & & 2 & 225 & 228,22 & $+3,22$ \\
\hline \multirow{4}{*}{ II } & \multirow{4}{*}{$\begin{array}{c}\text { Ouvrier } \\
\text { spécialisé } 2 \mathrm{e} \\
\text { degré }\end{array}$} & \multirow{4}{*}{$23,4 \%$} & 3 & 229 & 234,58 & $+5,58$ \\
\hline & & & 4 & 234 & 240,94 & $+6,94$ \\
\hline & & & 5 & 238,50 & 247,30 & $+8,80$ \\
\hline & & & 6 & 243,50 & 253,66 & $+10,16$ \\
\hline \multirow{5}{*}{ III } & \multirow{5}{*}{$\begin{array}{c}\text { Ouvrier } \\
\text { professionnel }\end{array}$} & \multirow{5}{*}{$49,6 \%$} & 7 & 247,50 & 260,02 & $+12,52$ \\
\hline & & & 8 & 252,50 & 266,38 & $+13,88$ \\
\hline & & & 9 & 257 & 272,74 & $+15,74$ \\
\hline & & & 10 & 263 & 279,10 & $+16,10$ \\
\hline & & & 11 & 268,50 & 285,46 & $+16,96$ \\
\hline \multirow{6}{*}{ IV } & \multirow{6}{*}{$\begin{array}{l}\text { Ouvrier qualifié } \\
1^{\text {er }} \text { degré }\end{array}$} & \multirow{6}{*}{$22 \%$} & 12 & 275 & 291,82 & $+16,82$ \\
\hline & & & 13 & 280,50 & 298,18 & $+17,68$ \\
\hline & & & 14 & 286,50 & 304,54 & $+18,04$ \\
\hline & & & 15 & 293,50 & 310,90 & $+17,40$ \\
\hline & & & 16 & 300,50 & 317,26 & $+16,76$ \\
\hline & & & 17 & 308,50 & 323,62 & $+15,12$ \\
\hline \multirow{7}{*}{ V } & \multirow{7}{*}{$\begin{array}{l}\text { Ouvrier qualifié } \\
2^{\mathrm{e}} \text { degré }\end{array}$} & \multirow{7}{*}{$2,6 \%$} & 18 & 316,50 & 329,98 & $+13,48$ \\
\hline & & & 19 & 325 & 336,34 & $+11,34$ \\
\hline & & & 20 & 333,50 & 342,70 & $+9,20$ \\
\hline & & & 21 & 341 & 349,06 & $+8,06$ \\
\hline & & & 22 & 350,50 & 355,42 & $+4,92$ \\
\hline & & & 23 & 362 & 361,78 & $-0,22$ \\
\hline & & & 24 & 374 & 368,14 & $-5,86$ \\
\hline
\end{tabular}

* en lires italiennes courantes.

Sources : CLSS AFIOM b. 48, «Chiarimenti in margine ad alcuni articoli dell'accordo ITALSIDER» [Clarifications de certaines clauses de l'accord ITALSIDER] [1961]; CLSS AFIOM. b. 49, Copie de la lettre du secrétariat national de la FIOM aux instances syndicales provinciales de Gênes, Piombino, Livourne, Naples, Trieste, Tarente et Novi Ligure (5 mai 1961).

Quelles sont les caractéristiques de ce nouveau système de classification et de rémunération? D'abord, il met fin aux lignes de partage antérieures entre professionnels et non professionnels : la catégorie de manœuvre disparaît au sein de celle, plus large, d'ouvrier spécialisé, qui est étendue sur deux niveaux comprenant six classes de rémunération. En outre, ce nouveau système classe plus finement les professionnels par l'ajustement du niveau de salaire en fonction du poste occupé et devient un outil privilégié de gestion du personnel. Ceci peut s'expliquer par la nature même de la cotation au poste, dont les critères d'évaluation se fondent, notamment, sur le degré de responsabilité requis par chaque poste : responsabilité des matériaux, de l'outillage et des machines, du travail, de la sécurité des autres salariés, etc. Les efforts demandés aux ouvriers vont ainsi dans le sens d'une valorisation de la maitrise des spécificités techniques du poste d'affectation, dont la définition est le produit des négociations entre la direction et les organisations syndicales, et non plus uniquement celui des opérations d'analyse effectuées par les ingénieurs des méthodes.

Il s'agit donc d'une politique visant la remobilisation du personnel par le biais d'une valorisation des salaires sur la base des postes que les ouvriers occupent, et qui vise essentiellement le groupe des professionnels (des ouvriers professionnels aux ouvriers qualifiés de $2^{\mathrm{e}}$ degré), à savoir les trois quarts des effectifs. Ceux-ci, en effet, bénéficient entre 1961 et 1962 d'une augmentation moyenne du salaire horaire de $12 \%$, le double de l'augmentation reçue par les ouvriers spécialisés (voir tableau 2 ). La construction de cette pyramide des classes de rémunérations relève d'un objectif de contrôle et d'homogénéisation des salariés que la mobilisation des groupes ouvriers contre le salaire au rendement n'a probablement fait qu'accélérer. Les effets de l'ancienneté du personnel ainsi que de la négociation collective ont probablement joué un rôle dans la détermination de ce résultat qui, au final, désamorce la rhétorique de la "déqualification ouvrière» due à l'innovation technologique et à la rationalisation des modes de gestion des salariés, argument mis en avant par la plupart des acteurs concernés bien qu'à partir de présupposés différents.

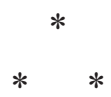

L'étude de l'application de la job evaluation dans l'usine italienne de Cornigliano permet d'éclairer comment cet outil de gestion a su répondre aux attentes du modèle de capitalisme qui s'affirme 
dans l'Europe de l'après-guerre et qui met l'accent sur l'exigence de marier efficience et équité afin de mobiliser les travailleurs et de stabiliser les relations sociales au sein des entreprises (BERLAND, Chiapello, 2004). Si les dynamiques internes à l'usine semblent donner raison à la politique de remobilisation des salariés poursuivie par la direction, il serait certainement peu généreux d'interpréter les comportements des travailleurs selon un modèle dichotomique, opposant la résistance à outrance à l'adhésion passive. La normalisation politique du personnel due au contexte de guerre froide et à la bataille anti-communiste, comme le retour des organisations syndicales hostiles aux politiques de la direction dans le jeu de la négociation collective grâce à la mobilisation des groupes ouvriers, sont autant d'éléments qui contribuent à rejeter une vision trop linéaire de l'histoire.

Ce sont finalement les dirigeants de l'entreprise génoise qui dessinent un portrait en demi-teinte des résultats issus de la mise en œuvre du salaire au poste. Interpellés sur la question dans le cadre des enquêtes sociologiques de la CECA évoquées plus haut, ils n'hésitent guère à souligner les attentes déçues liées à cet outil de gestion du personnel, en matière notamment de négociation des augmentations salariales, qui dépend désormais des mécanismes «objectifs» de la cotation au poste, ou d'avancement de carrière, car «pour avancer il faut tout de même changer de métier» ${ }^{(51)}$.

Ainsi, le projet de régulation sociale préconisé par les experts de la CECA, qui voient dans la rationalisation des modes de gestion du personnel ouvrier un moyen pour harmoniser à la fois les relations sociales et les conditions de travail dans le nouvel espace communautaire, ressort fortement amendé. En témoigne, par exemple, la persistance des hiérarchies professionnelles issues des accords nationaux ou locaux. Celles-ci sont l'expression de conventions et de jugements de valeur ancrés dans des contextes et traditions spécifiques (SAGLIO, 1987), et donnent lieu à des formes hybrides de classifications d'emplois faisant la part entre, d'un côté, la visée rationalisatrice des experts en organisation et, de l'autre côté, le besoin de reconnaissance de l'expérience professionnelle ressenti par nombre d'ouvriers. Un tel ensemble de facteurs incite, à l'instar de ce que font les historiens et anthropologues des techniques, à appréhender les outils de gestion dans leur dimension située, voire localisée (Latour, 1989; Perez, Verna, 2009). Si la job evaluation fait l'objet d'un processus de circulation, celui-ci relève aussi bien du transfert international que de l'interaction avec des pratiques situées à l'échelle locale, qui contribuent à façonner le «sens » qu'on prête à cet outil (COHEN, RAJ, 2007).

\section{Bibliographie}

Berland N., Chiapello E. (2004), «Le rôle des réformateurs sociaux dans la diffusion de nouvelles pratiques de gestion : le cas du contrôle budgétaire en France (1930-1960)», Comptabilité-Contrôle-Audit, juin, pp. 133-160.

BIT (Bureau international du travail) (1960), La qualification du travail, Genève, Imprimerie Granchamp.

Boltanski L. (1981), «America America... Le plan Marshall et l'importation du "management"», Actes de la recherche en sciences sociales, $n^{\circ} 38$, pp. 19-41.

Boltanski L., Chiapello E. (1999), Le nouvel esprit du capitalisme, Paris, Gallimard.

CAREW A. (1987), Labor under the Marshall plan. The politics of productivity and the marketing of the management science, Manchester, Manchester University Press.

(51) AHUE BAC 10/1969-5, «Nota relativa alla ricerca sociologica sui sistemi di retribuzione della siderurgia italiana; Interviste con dirigenti dell'ITALSIDER» [Rapport concernant la recherche sociologique sur les systèmes de rétribution de la sidérurgie italienne; entretiens avec les dirigeants de la société ITALSIDER] (mars-mai 1962), pp. 7 et 37.
CECA (Communauté européenne du charbon et de l'acier. Haute autorité) (1957), La qualification du travail (job evaluation) et ses applications dans la sidérurgie de la Communauté. Italie, Luxembourg, Service de publication des communautés européennes.

CECA (Communauté européenne du charbon et de l'acier. Haute autorité) (1963), C.E.C.A 1952-1962. Résultats, limites, perspectives, Luxembourg, Service de publication des communautés européennes.

Cohen Y., RaJ K. (2007), «Histoire des circulations : quelques réflexions », EHESS, document de travail.

Dadoy M. (1973), «Les systèmes d'évaluation de la qualification du travail. Pratique et idéologie», Sociologie du travail, $\mathrm{n}^{\circ} 2$, pp. 115-135.

Dofny J., Durand C., Reynaud J.-D., Touraine A. (1966), Les ouvriers et le progrès technique. Étude d'un cas : un nouveau laminoir, Paris, Armand Colin.

DORIA M. (2004-2005), «La siderurgia a Genova nel secondo dopoguerra. Nuovi investimenti, ristrutturazioni, lotte operaie», Annali di storia dell'impresa, $\mathrm{n}^{\circ}$ 15-16, pp. 75-106. 
Durand C., Prestat C., Willener A. (1972), Travail, salaire, production. Le contrôle des cadences, Tome I, Paris, Mouton.

FAscetti A. (1959), Discorso ai dipendenti della Cornigliano sullo sviluppo della siderurgia e sui rapporti sindacali. Genova - Cornigliano, 5 agosto 1959, Rome, Edindustria.

Felisini D. (2013), «Biografie di un gruppo dirigente (1945-1970)», in Amatori F. (dir.), Storia dell'IRI. 2. Il " miracolo» economico e il ruolo dell'IRI, Rome-Bari, Laterza, pp. 152-258.

Giugni G. (1953), Mansioni e qualifiche nel rapporto di lavoro, Naples, Jovene.

JoIn-LAmbert O., Lochard Y. (2010), «Construire le mérite dans la fonction publique d'État. Le cas de la Culture », Sociologie du travail, vol. 53, n² 2, pp. 38-56.

IPSOA (Terzo Gruppo di Studio su problemi di Relazioni Umane)(1955-1956), Organizzazione e razionalizzazione del lavoro presso alcuni enti del gruppo Finsider, s. n. t.

IRI (Servizio problemi del lavoro) (1960), Aspetti giuridico-contrattuali dell'introduzione della job evaluation, s. n. t.

IRI (Servizio problemi del lavoro) (1962), I sindacati dei lavoratori e la job evaluation, s. n. t., février.

Latour B. (1989), La science en action, Paris, La Découverte.

LomBA C. (2001), L'incertitude stratégique au quotidien : trajectoire d'entreprises et pratiques de travail. Le cas de l'entreprise sidérurgique Cockerill Sambre, 1970-1998, Thèse en sociologie, EHESS.

Lomвa C. (2004), «Distinguer un ouvrier d'un employé dans l'industrie : naturalisation et négociations des classifications», Sociétés contemporaines, $\mathrm{n}^{\circ}$ 54, pp. 35-53.

Lutz B., Willener A. (1960), Niveau de mécanisation et mode de rémunération, Luxembourg, Service de publication des communautés européennes.

MAIER C.S. (1987), «The politics of productivity : foundations of American international economic policy after World War II », in Maier C.S., In search of stability. Explorations in historical political economy, Cambridge, Cambridge University Press, pp. 153-184.

MeChi L. (2004), «Le politiche sociali della CECA», in Ranieri R., Tosi L. (dir.), La Comunità Europea del
Carbone e dell'Acciaio (1959-2002). Gli esiti del trattato in Europa e in Italia, Padoue, Cedam, pp. 105-126.

Mioche (2004), «La CECA e la ristrutturazione europea del mercato siderurgico », in Ranieri R., Tosi L. (dir.), La Comunità Europea del Carbone e dell'Acciaio (19592002). Gli esiti del trattato in Europa e in Italia, Padoue, Cedam, pp. 85-103.

Mottez B. (1966), Systèmes de salaire et politiques patronales. Essai sur l'évolution des pratiques et des idéologies patronales, Paris, Éditions du CNRS.

Moutet A. (1997), Les logiques de l'entreprise. La rationalisation dans l'industrie française de l'entredeux-guerres, Paris, Éditions de l'EHESS.

Moutet A. (2005), «Études de temps et intensification du travail dans l'industrie française de 1945 à la décennie $1960 »$, in Linhart D., Moutet A. (dir.), Le travail nous est compté. La construction des normes temporelles $d u$ travail, Paris, La Découverte, pp. 28-62.

Pérez L., Verna C. (2009), «La circulation des savoirs techniques du Moyen Âge à l'époque moderne. Nouvelles approches et enjeux méthodologiques», Tracés. Revue de sciences humaines, $\mathrm{n}^{\circ} 16$, pp. 25-61.

Pezet É. (2000), «Négociation collective et gouvernement des individus dans l'entreprise : la négociation des classifications dans la métallurgie (1968-1975)», Entreprises et Histoire, ${ }^{\circ} 26$, pp. 75-88.

Quaid M. (1993), Job evaluation: the myth of the equitable assessment, Toronto, Toronto University Press.

RICCIARDI F. (2010), «The circulation of practices. Americanizing social relations at the Cornigliano steel plant (Italy), 1948-1960», Labor History, vol. 51, n 2 , pp. 170-182.

Rosental P.-A. (2006), «Géopolitique et Étatprovidence : le BIT et la politique mondiale des migrations dans l'entre-deux-guerres », Annales. Histoire, sciences sociales, vol. 61, n 1, pp. 99-134.

SAGLio J. (1987), «Hiérarchie des salaires et négociation de classifications. France 1900-1950», Travail et emploi, $\mathrm{n}^{\circ} 27, \mathrm{pp} .7-19$.

Steinhouse A. (2006), «La participation des travailleurs dans la France de l'après-Seconde guerre mondiale. Éléments d'une comparaison internationale», in Chatriot A., Join-Lambert O., Viet V. (dir.), Les politiques $d u$ Travail (1906-2006). Acteurs, institutions, réseaux, Rennes, Presses universitaires de Rennes, pp. 415-427. 